\title{
Hepatotoxicidad inducida por glucosamina- condroitina en un hospital público de Lima (Perú): reporte de caso
}

\section{Case report of glucosamine-chondroitin induced hepatotoxicity in a public hospital in Lima, Peru}

Walter Zagaceta Torres, MD,$^{{ }^{*}}$ Jorge Luis Garavito Rentería, MD. ${ }^{2}$

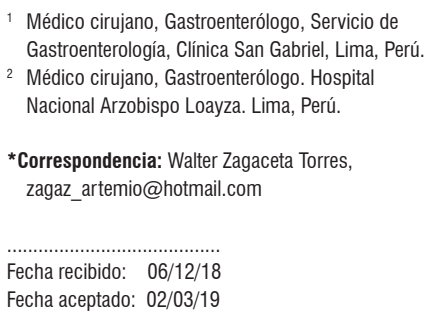

\begin{abstract}
Resumen
En el cuerpo humano tenemos glucosamina y condroitina de forma natural. Estas sustancias constituyen un componente importante del sistema cartilaginoso. Como medicamentos, tienen múltiples indicaciones clínicas, principalmente la osteoartritis. La hepatotoxicidad inducida por estas biomoléculas es infrecuente, pues cuentan solo con reportes de casos aislados en la literatura mundial. En este trabajo, presentamos el caso de una paciente con una lesión hepática inducida por glucosamina-condroitina del tipo hepatocelular, que fue admitida en el hospital por causa de una sintomatología respiratoria y malestar general. En ella, se destacó una marcada hipertransaminasemia durante los exámenes de laboratorio. Asimismo, se descartaron etiologías como el alcohol, hepatitis virales y hepatopatías autoinmunes, principalmente. De igual forma, no se llegó a evidenciar una enfermedad hepática crónica mediante la ecografía abdominal. Al suspenderse el medicamento, se observó una disminución considerable de la hipertransaminasemia luego de 1 semana, y una mejoría total de esta a los 2 meses del alta hospitalaria. Este caso se añade a los pocos reportados a nivel mundial y cobra una importancia relevante para la publicación de posteriores estudios sistemáticos que aclaren el panorama de esta enfermedad.
\end{abstract}

\section{Palabras clave}

Glucosamina, condroitina, toxicidad, transaminasas, osteoartritis.

\begin{abstract}
The human body naturally produces glucosamine and chondroitin which are important components of the cartilaginous system. There are multiple clinical indications for them as medicines, but they are primarily used for osteoarthritis. Hepatotoxicity induced by these biomolecules is uncommon, and the only reports in the world literature are isolated individual cases. This article presents the case of a patient with glucosaminechondroitin-induced hepatocellular damage who was admitted to the hospital with respiratory symptoms and malaise. Marked hypertransaminemia was found in laboratory tests. Etiologies such as alcohol, viral hepatitis and autoimmune liver diseases were ruled out, and abdominal ultrasound found no evidence of chronic liver disease. Discontinuance of glucosamine and chondroitin led to a considerable decrease in hypertransaminemia after one week with total improvement two months of hospital discharge. This case adds to the small number reported worldwide and is relevant for future systematic studies to clarify the outlook for this disease.
\end{abstract}

\section{Keywords}

Glucosamine, chondroitin, toxicity, transaminases, osteoarthritis. 


\section{INTRODUCCIÓN}

Las biomoléculas glucosamina y condroitina, como componentes naturales del cuerpo humano, son vitales para la estructura del sistema cartilaginoso, pero aplicadas en forma exógena pueden tener efectos tanto favorables, como perjudiciales.

Aunque actualmente en Perú, la Dirección General de Medicamentos, Insumos y Drogas (DIGEMID) no justifica su uso para el tratamiento de osteoartritis, dado su alto costo (1), en algunos centros de Estados Unidos y el Reino Unido se ha aprobado su prescripción, en vista de la mayor repercusión de sus efectos beneficiosos (2).

Sin embargo, a pesar de su aparente inocuidad, su uso no está exento de efectos adversos, tales como molestias gastrointestinales, somnolencia, alteraciones dermatológicas, cefalea e incluso puede llegar a producir eventos cardiovasculares (3).

Asimismo, se pueden producir alteraciones bioquímicas como la presentación de una lesión hepática en las formas hepatocelular, colestásica y mixta, con repercusiones tanto clínicas como bioquímicas. En efecto, su uso puede conducir a situaciones extremas como una falla hepática fulminante y la muerte. Estos desenlaces ya han sido reportados en varios casos y algunos de ellos han sido demostrados mediante una biopsia hepática (4-10).

Presentamos el caso de una paciente que tuvo una lesión hepática con patrón hepatocelular, inducida por la glucosamina-condroitina, en un hospital público de Lima (Perú).

\section{PRESENTACIÓN DEL CASO}

Paciente mujer de 65 años, natural de Abancay, con antecedente de osteoartritis en la rodilla derecha. Desde hace 1 año, la paciente se encuentra en tratamiento con naproxeno, en forma irregular, mientras que el tratamiento esporádico para el asma crónica se realiza con salbutamol y con el uso reciente de un medicamento comercial basado en glucosamina-condroitina, por 10 días. La paciente niega la ingesta de alcohol y de productos herbáceos, así como antecedentes familiares de importancia.

La mujer acude a emergencia dado que presenta un tiempo de enfermedad de 1 día de evolución, con un cuadro clínico caracterizado por dolor torácico asociado a malestar general y 3 episodios de hemoptisis, en un volumen total aproximado de $300 \mathrm{~cm}^{3}$. Durante el examen físico en emergencia, luce ligeramente pálida y en un aparente regular estado general, pero hemodinámicamente estable.

No se aprecian estigmas de hepatopatía crónica y no hay signos de visceromegalia, ni adenopatías. Por tanto, se realiza una tomografía espiral multicorte de tórax con contraste y se diagnostican bronquiectasias y enfermedad pulmonar intersticial difusa.

Los exámenes de laboratorio de emergencia muestran los siguientes resultados:

- Hemoglobina ( $\mathrm{Hb}): 11,3$

- Leucocitos: 3940

- Plaquetas: 191000

- Transaminasa glutámica-oxalacética (TGO): 672

- Transaminasa glutámico-pirúvica (TGP): 689

- Bilirrubina total (BT): 0,71

- Bilirrubina directa (BD): 0,38

- Proteínas totales (PT): 8,21

- Albúmina (Alb): 4,2

- Fosfatasa alcalina (FA): 160

- Gamma-glutamil transpeptidasa (GGT): 244

- Tiempo de protrombina (TP): 14,2

- Índice internacional normalizado (International Normalized Ratio, INR): 1,2

- Creatina-fosfocinasa (Creatine Phosphokinase, CPK) total: 136

- CPK-MB: 24

- Lactato deshidrogenasa (LDH): 764

- Virus de la inmunodeficiencia humana (VIH): no reactivo

- Ferritina: 124,5

- T4 libre: 1,51

- Hormona estimulante de la tiroides (ThyroidStimulating Hormone, TSH): 3,36

Entre tanto, los marcadores virales para hepatitis A, B, C, $D$ y $E$ fueron negativos, mientras que los marcadores de los anticuerpos antinucleares (Antinuclear Antibodies, ANA), antimúsculo liso (Anti-Smooth Muscle Antibodies, ASMA), antimitocondriales serotipo M2 (Anti-mitochondrial Antibodies, AMA M2), antimicrosomal hígado/riñón (Liver Kidney Microsome Antibodies, LKM-1) y anticitoplasma de neutrófilos (Antineutrophil Cytoplasmic Antibody, ANCA) fueron negativos. La inmunoglobulina G marcó 1842 y la inmunoglobulina $\mathrm{M}$ estuvo en 31,7 (dentro de los rangos normales).

La ecografía abdominal durante la hospitalización mostró la existencia de barro biliar y meteorismo intestinal incrementado; hígado de tamaño normal; bordes uniformes y parénquima homogéneo. No se evidenciaron lesiones focales ni difusas. Además, las vías biliares intrahepáticas no se observaron dilatadas. El colédoco y la vena porta tuvieron un calibre normal.

La paciente es dada de alta, luego de haber mejorado su proceso respiratorio dos semanas después. Se indicaron medicamentos para la mejoría de su enfermedad de fondo, así como también se sugirió la no ingesta de glucosamina- 
condroitina, sustancias que dejó de consumir unos días antes de la admisión en el hospital.

Después de 2 meses del alta, la paciente acude al hospital y muestra una mejoría clínica. Sus controles de laboratorio presentan los siguientes resultados:

- Hb: 12,1

- Hematocrito (Hcto): $36,2 \%$

- Leucocitos: 4260

- Ab: $0 \%$

- Plaquetas: 208000

- TP: 14,7

- INR: 1,31

- TGO: 37

- TGP: 25

- BT: 0,57

- BD: 0,24

- FA: 223

- GGT: 57

- PT: 7,29

- Alb: 4,06

En la Tabla 1 se describen los datos de la bioquímica hepática de la paciente.

Tabla 1. Resultados de los exámenes de biometría hepática de la paciente con hepatotoxicidad por glucosamina-condroitina

\begin{tabular}{lcccc}
\hline & $\begin{array}{c}\text { En el momento } \\
\text { de la admisión }\end{array}$ & $\begin{array}{c}\text { 2 días } \\
\text { después }\end{array}$ & $\begin{array}{c}\text { 1 semana } \\
\text { después }\end{array}$ & $\begin{array}{c}\text { 2 meses } \\
\text { después }\end{array}$ \\
\hline TGO (UI) & 672 & 296 & 388 & 37 \\
TGP $(\mathrm{UI})$ & 689 & 392 & 383 & 25 \\
$\mathrm{BT}(\mathrm{mg} / \mathrm{dL})$ & 0,71 & 1,69 & 2,82 & 0,57 \\
$\mathrm{BD}(\mathrm{mg} / \mathrm{dL})$ & 0,38 & 1,47 & 2,24 & 0,24 \\
$\mathrm{PT}(\mathrm{g} / \mathrm{dL})$ & 8,21 & 6,1 & 7,1 & 7,29 \\
ALB $(\mathrm{g} / \mathrm{dL})$ & 4,2 & 3,1 & 3,5 & 4,06 \\
FA $(\mathrm{UI})$ & 160 & 104 & 101 & 73 \\
$\mathrm{GGT}(\mathrm{UI})$ & & 212 & 198 & 57 \\
TP $(\mathrm{s})$ & 14,2 & & & 14,7 \\
INR & 1,2 & & & 1,31 \\
\hline
\end{tabular}

TGO: transaminasa glutámico-oxalacética (valor negativo [VN]: 0-35 UI); TGP: transaminasa glutámico-pirúvica (VN: 0-35 UI); BT: bilirrubina total (VN: 0,3-1,2 mg/dL); BD: bilirrubina directa (VN: 0-0,3 mg/dL); PT: proteínas totales (VN: 6-8 g/dL); ALB: albúmina (VN: 3,5-5,5 g/dL); FA: fosfatasa alcalina (36-129 UI); GGT: gammaglutamil transpeptidasa (VN: 8-78 UI); TP: tiempo de protrombina (VN: 10-14 s); INR: índice internacional normalizado (International Normalized Ratio) (VN: $<1,4)$

\section{DISCUSIÓN}

La glucosamina y la condroitina son biomoléculas que se pueden encontrar en forma natural dentro del cuerpo humano y mantienen la integridad del sistema cartilaginoso, a través de múltiples procesos (11).

La glucosamina es un componente de la familia de los glicosaminoglicanos que se encuentra en la matriz cartilaginosa y en el líquido sinovial. Puede tener efectos medicinales cuando se administra externamente (12), sobre todo en enfermedades osteoarticulares como la osteoartritis, en la cual se ha comprobado su efectividad y seguridad para mitigar los síntomas (13). Sin embargo, no se ha demostrado su uso como profilaxis para evitar la aparición de la osteoartritis (14).

El caso descrito se trata de una paciente que tuvo un daño hepático del tipo hepatocelular, directamente relacionado con la ingesta de un medicamento comercial basado en glucosamina-condroitina. Se menciona que el tiempo de inicio de las alteraciones hepáticas oscila entre 1 y 4 semanas, luego de iniciar la ingesta del medicamento, y su patrón de lesión es típicamente hepatocelular, como en nuestro caso, o mixto. El mecanismo por el cual se produce dicha hepatotoxicidad aún permanece desconocido (15).

Es importante recalcar que la paciente en ningún momento presentó un cuadro clínico asociado con hepatopatía como astenia, prurito, ictericia y náuseas. En contraste, Smith y Dillon (4) publicaron tres casos de hepatopatía en pacientes que consumieron preparados herbáceos que contenían glucosamina: dos de ellos llegaron a tener un daño hepatocelular y uno derivó en una falla hepática fulminante y la muerte.

Dos de los tres pacientes tuvieron un cuadro clínico asociado con hepatopatía (ictericia y prurito), los cuales mostraron una mejoría (al igual que la bioquímica hepática), luego de retirar los suplementos herbáceos. Asimismo, el paciente que no mostró sintomatología tuvo una remisión bioquímica al retirar dichos suplementos.

Ebrahim y colaboradores (5) reportaron a una paciente con malestar general e ictericia y a partir de sus exámenes de laboratorio revelaron que tenía un patrón mixto de lesión hepática (hepatocelular y colestásica). Estas alteraciones fueron asociadas a la ingesta de suplementos de glucosamina dos semanas previas a la admisión. La mejoría clínica y bioquímica se vio luego de una semana de retirar el medicamento y en cuatro semanas se normalizó completamente.

Estos hechos son similares a los de nuestra paciente, que dejó de tomar el medicamento unos días antes de ingresar al hospital, y posterior a ello, se observó que los valores disminuyeron hasta en un $50 \%$ (con respecto a las transaminasas); luego de ocho semanas volvieron a la normalidad.

Por su parte, Yang y colaboradores (6) reportaron el caso de una paciente que tuvo un daño hepático mixto luego de consumir un producto comercial que contenía glucosamina-condroitina y productos herbáceos. Este evento es similar al nuestro, con una exposición a glucosaminacondroitina. 
Un punto importante para dicho caso fue la demostración, mediante biopsia, del daño hepático, lo cual no se pudo realizar en nuestro estudio, ya que la paciente no lo requirió por presentarse asintomática. Del mismo modo, Linnebur y colaboradores (7) demostraron la asociación de dicho producto comercial y el daño hepático, a partir del reporte de dos casos de hepatotoxicidad relacionada con glucosamina-condroitina.

De otro lado, Ossendza y colaboradores (8) describieron un caso con elevación de transaminasas y bilirrubina (daño hepatocelular), traducidas clínicamente en prurito e ictericia, asociadas a la ingesta en dosis terapéuticas de glucosamina, que remitieron luego de retirar el medicamento. Es importante recalcar que nuestra paciente, en cierto momento de la evolución, comenzó a presentar un incremento de la bilirrubina.

Asimismo, podemos mencionar el caso único reportado por Stephen y colaboradores (9), el cual se trató de un paciente que se presentó con ictericia y colestasis, cuya etiología fue incierta durante tres meses de estudio. Posteriormente, se determinó la asociación directa de sus síntomas con la ingesta de glucosamina-condroitina y se demostró una lesión colestásica por medio de una biopsia hepática.

Es importante mencionar el trabajo desarrollado por Cerda y colaboradores (10), el cual estuvo basado en la realización de un cuestionario a 151 pacientes con enfermedad hepática crónica subyacente, a fin de determinar la asociación de la ingesta de glucosamina-condroitina y las alteraciones de las pruebas hepáticas. Los autores lograron establecer que dos pacientes (uno de ellos incluyó un cuadro clínico) tuvieron alteraciones en las transaminasas y en las enzimas de colestasis asociadas con el medicamento.

En síntesis, nuestro paciente se suma a la estadística de los pocos casos registrados hasta el momento de hepatotoxicidad inducida por glucosamina-condroitina. En efecto, este es el primer caso reportado en Perú.

Por tanto, recomendamos seguir investigando las etiologías de todas las alteraciones en las pruebas hepáticas de nuestros pacientes que llegan a la admisión hospitalaria, tanto en emergencias como en la consulta externa y la hospitalización. Solo así podremos incrementar la casuística de la lesión hepática inducida por drogas y tener un mejor panorama acerca de esta creciente patología.

\section{REFERENCIAS}

1. Ministerio de Salud. Informe técnico $N^{\circ} 22-2007$ : glucosamina sulfato 1,5 g/condroitina 1,2 g (polvo). Lima: Dirección General de Medicamentos, Insumos y Drogas; 2007.

2. Hochberg MC, Martel-Pelletier J, Monfort J, Möller I, Castillo JR, Arden N, et al. Combined chondroitin sulfate and glucosamine for painful knee osteoarthritis: a multi- centre, randomised, double-blind, non-inferiority trial versus celecoxib. Ann Rheum Dis. 2016;75(1):37-44. http:// dx.doi.org/10.1136/annrheumdis-2014-206792

3. Sawitzke AD, Shi H, Finco MF, Dunlop DD, Harris CL, Singer NG, et al. Clinical efficacy and safety of glucosamine, chondroitin sulphate, their combination, celecoxib or placebo taken to treat osteoarthritis of the knee: 2-year results from GAIT. Ann Rheum Dis. 2010;69(8):1459-64. http:// dx.doi.org/10.1136/ard.2009.120469

4. Smith A, Dillon J. Acute liver injury associated with the use of herbal preparations containing glucosamine: three case studies. BMJ Case Rep. 2009;2009. pii: bcr02.2009.1603. https://doi.org/10.1136/bcr.02.2009.1603

5. Ebrahim V, Albeldawi M, Chiang DJ. Acute liver injury associated with glucosamine dietary supplement. BMJ Case Rep. 2012;2012. pii: bcr2012007665. https://doi.org/10.1136/ bcr-2012-007665

6. Yang L, Aronsohn A, Hart J, Jensen D. Herbal hepatoxicity from Chinese skullcap: A case report. World J Hepatol. 2012;4(7):231-233. https://doi.org/10.4254/wjh.v4.i7.231

7. Linnebur SA, Rapacchietta OC, Vejar M. Hepatotoxicity associated with chinese skullcap contained in Move Free Advanced dietary supplement: two case reports and review of the literature. Pharmacotherapy. 2010;30(7):750, 258e-262e. https://doi.org/10.1592/phco.30.7.750

8. Ossendza RA, Grandval P, Chinoune F, Rocher F, Chapel F, Bernardini D. Acute cholestatic hepatitis due to glucosamine forte. Gastroenterol Clin Biol. 2007;31(4):449-50. https://doi.org/10.1016/S0399-8320(07)89410-3

9. Ip S, Jeong R, Schaeffer DF, Yoshida EM. Unusual case of drug-induced cholestasis due to glucosamine and chondroitin sulfate. World J Hepatol. 2015;7(24):2559-2562. https://doi.org/10.4254/wjh.v7.i24.2559

10. Cerda C, Bruguera M, Parés A. Hepatotoxicity associated with glucosamine and chondroitin sulfate in patients with chronic liver disease. World J Gastroenterol. 2013;19(32):5381-4. https://doi.org/10.3748/wjg.v19.i32.5381

11. Fransen M, Agaliotis M, Nairn L, Votrubec M, Bridgett L, $\mathrm{Su} S$, et al. Glucosamine and chondroitin for knee osteoarthritis: a double-blind randomized placebo-controlled clinical trial evaluating single and combination regimens. Ann Rheum Dis. 2015;74(5):851-8. https://doi.org/10.1136/ annrheumdis-2013-203954

12. Bruyère $\mathrm{O}$, Altman $\mathrm{RD}$, Reginster JY. Efficacy and safety of glucosamine sulfate in the management of osteoarthritis: Evidence from real-life setting trials and surveys. Semin Arthritis Rheum. 2016;45(4 Suppl):S12-7. https://doi. org/10.1016/j.semarthrit.2015.11.011

13. Clegg DO, Reda DJ, Harris CL, Klein MA, O’Dell JR, Hooper MM, et al. Glucosamine, chondroitin sulfate, and the two in combination for painful knee osteoarthritis. N Engl J Med. 2006;354(8):795-808. https://doi. org/10.1056/NEJMoa052771

14. de Vos BC, Landsmeer MLA, van Middelkoop M, Oei EHG, Krul M, Bierma-Zeinstra SMA, et al. Long-term effects of a lifestyle intervention and oral glucosamine sulphate in 
primary care on incident knee OA in overweight women. Rheumatology (Oxford). 2017;56(8):1326-1334. https:// doi.org/10.1093/rheumatology/kex145

15. LiverTox: Clinical and Research Information on DrugInduced Liver Injury [Internet]. Bethesda (MD): National
Institute of Diabetes and Digestive and Kidney Diseases; 2012. Glucosamine. [actualizada el 12 de marzo de 2020]. Disponible en: https://www.ncbi.nlm.nih.gov/books/ NBK547949/ 